\title{
Mobilité et lutte contre la pollution atmosphérique
} La difficile conciliation des exigences environnementales et de l'équité sociale dans l'instauration d'une zone à basse émission

\section{Mobility and air pollution}

The difficult conciliation between environmental constraints and social equity in the introduction of low emission zone

Movilidad y lucha contra la contaminación atmosférica La difícil conciliación entre las exigencias ambientales y la

\section{Julie Gobert}

\section{Volume 57, numéro 161, septembre 2013}

Version originale soumise en mars 2013. Version révisée reçue en septembre 2013.

URI : https://id.erudit.org/iderudit/1024905ar

DOI : https://doi.org/10.7202/1024905ar

Aller au sommaire du numéro

Éditeur(s)

Département de géographie de l’Université Laval

ISSN

0007-9766 (imprimé)

1708-8968 (numérique)

Découvrir la revue

Citer cet article

Gobert, J. (2013). Mobilité et lutte contre la pollution atmosphérique : la difficile conciliation des exigences environnementales et de l'équité sociale dans l'instauration d'une zone à basse émission. Cahiers de géographie du Québec, 57(161), 277-298. https://doi.org/10.7202/1024905ar

\section{Résumé de l'article}

Dans un but de développement économique des territoires, les pouvoirs publics sont amenés, en France, à favoriser le « capital mobilité » des individus pour leur donner le moyen d'accéder au marché du travail et à un ensemble de ressources et de services. Toutefois, il est également nécessaire de réguler les problèmes liés à l'automobilité et aux nuisances environnementales qui lui sont inhérentes. Les politiques publiques de transport ont de ce fait évolué : la notion de " droit à la mobilité » se dissout peu à peu au profit d'objectifs plus complexes de développement durable. Ce dilemme entre enjeux économiques, sociaux et environnementaux pose la question des inégalités environnementales, particulièrement quand il s'agit de mettre en place une zone à basse émission sur les grandes agglomérations, comme cela est prévu dans la loi du Grenelle II sous le nom de zone d'actions prioritaires pour l'air (ZAPA). Au regard d'une enquête de terrain menée sur une intercommunalité de Seine-Saint-Denis, en amont de la mise en place d'une ZAPA, nous avons cherché à mieux connaître l'impact d'une telle mesure et les possibles répercussions en termes d'inégalités.
Tous droits réservés @ Cahiers de géographie du Québec, 2013

Ce document est protégé par la loi sur le droit d'auteur. L’utilisation des services d'Érudit (y compris la reproduction) est assujettie à sa politique d'utilisation que vous pouvez consulter en ligne.

https://apropos.erudit.org/fr/usagers/politique-dutilisation/ 


\title{
Mobilité et lutte contre la pollution atmosphérique.
}

\section{La difficile conciliation des exigences environnementales et de l'équité sociale dans l'instauration d'une zone à basse émission}

\author{
Mobility and air pollution. The difficult \\ conciliation between environmental \\ constraints and social equity in the \\ introduction of low emission zone \\ Movilidad y lucha contra la contaminación \\ atmosférica. La difícil conciliación entre las \\ exigencias ambientales y la equidad social \\ en la instauración de una zona de baja \\ emisión
}

\author{
Julie GOBERT \\ Centre de Recherches et d'Études \\ Interdisciplinaires sur le Développement \\ Durable - Université de technologie de Troyes \\ Julie.Gobert@gmail.com
}

\begin{abstract}
Résumé
Dans un but de développement économique des territoires, les pouvoirs publics sont amenés, en France, à favoriser le "capital mobilité» des individus pour leur donner le moyen d'accéder au marché du travail et à un ensemble de ressources et de services. Toutefois, il est également nécessaire de réguler les problèmes liés à l'automobilité et aux nuisances environnementales qui lui sont inhérentes. Les politiques publiques de transport ont de ce fait évolué: la notion de «droit à la mobilité» se dissout peu à peu au profit d'objectifs plus complexes de développement durable. Ce dilemme entre enjeux économiques, sociaux et environnementaux pose la question des inégalités environnementales, particulièrement quand il s'agit de mettre en place une zone à basse émission sur les grandes agglomérations, comme cela est prévu dans la loi du Grenelle II sous le nom de zone d'actions prioritaires pour l'air (ZAPA). Au regard d'une enquête de terrain menée sur une intercommunalité de Seine-Saint-Denis, en amont de la mise en place d'une ZAPA, nous avons cherché à mieux connaître l'impact d'une telle mesure et les possibles répercussions en termes d’inégalités.
\end{abstract}

\section{Mots-clés}

Mobilité, inégalités environnementales, zone à basse émission, droit à un environnement sain, sentiment de justice, équité. 


\begin{abstract}
In the interest of economic development, local and national authorities in France are finding themselves confronted with the need to adopt public policies designed to encourage mobility and improve access to job opportunities and public services. At the same time, it is becoming increasingly important to reduce motor vehicle traffic, this being one of the main causes of environmental damage, pollution and health problems. Public policy decisions relating to transport are evolving; the previous key issue of the right to mobility is gradually giving way to more complex goals pertaining to sustainable development. Achieving the difficult balance between economic, social and environmental objectives brings to the fore the question of environmental imbalances. This became a critical issue when it was decided to implement Low Emission Zones (ZAPA) in major urban areas in France. We carried out a field study on the community of Seine-Saint-Denis to better understand the different impacts a ZAPA can have on a local population.
\end{abstract}

\title{
Keywords
}

Mobility, Low Emission Zones, environmental imbalances, right to a healthy environment, perceived fairness, equity.

\section{Resumen}

Dentro del desarrollo económico de territorios, los poderes públicos de Francia son incitados a favorecer el "capital movilidad" de los individuos fin de darles medios de acceso al mercado de trabajo y a un conjunto de servicios. Es igualmente necesario normalizar los problemas del automovilismo y de los ruidos ambientales inherentes. Las políticas públicas de transporte han evolucionado: la noción de "derecho a la movilidad" se disuelve poco a poco, pro objetivos complejos de desarrollo sostenido (lominio y limitación del flujo de motorizados para disminuir la contaminación atmosférica) . El dilema entre contingencias económicas, sociales y ambientales plantea el problema de las desigualdades ambientales, especialmente cuando se trata de crear una zona a baja emisión sobre grandes aglomeraciones, como previsto en la ley de Grenelle II, llamada Zona de Acciones Prioritarias del Aire (ZAPA). Concerniendo un estudio de terreno en una inter-comunidad de Seine-Saint-Denis, precediendo una ZAPA, hemos tratado de conocer mejor el impacto de tal medida y de sus repercusiones sobre la desigualdad.

\section{Palabras claves}

Movilidad, desigualdades ambientales, zona a baja emisión. Derecho a un ambiente sano, sentimiento de justicia. Equidad.

\section{Introduction}

Les inégalités environnementales constituent une problématique émergente dans le contexte français. Après quelques hésitations terminologiques («environnementales» vs «écologiques») (Emelianoff, 2006; Chaumel et La Branche, 2008), les chercheurs s'approprient peu à peu la question. Celle-ci reste à l'inverse peu courante dans la société civile (associations, syndicats...) et les pouvoirs publics, sauf sur l'aspect de la précarité énergétique. Certes, différents programmes de recherche commencent à être financés par le Centre national de la recherche scientifique (CNRS) ou le ministère 
responsable du développement durable pour définir, observer et objectiver de possibles situations d'inégalités environnementales dans le contexte français, mais sans que cela engendre un débat public.

Les questionnements autour des conséquences sanitaires de l'exposition à un environnement de mauvaise qualité ont cependant reçu plus d'échos. Les préoccupations concernant la santé sont en effet devenues un levier fondamental des experts et des associations pour interpeller les pouvoirs publics sur des projets locaux ou des situations critiques. Mais toutes les pollutions ne suscitent pas la même mobilisation; de fait, elles créent d'autant plus d'inquiétude et de résistance qu'un «coupable» est identifiable (usine déversant des déchets dans la nature, volutes blanches sortant de la cheminée d'une industrie) et non une multiplicité dans laquelle chacun peut se reconnaître (les émissions des véhicules). Or la pollution atmosphérique, parce qu'elle est diffuse, peu visible et qu'a priori ses effets s'étalent dans le temps, a longtemps bénéficié d'une non-prise de conscience, si ce n'est par le biais de l'hygiénisme (Charles, 2009). Pourtant, plusieurs études qualitatives et quantitatives longitudinales de cohortes viennent corroborer un lien de cause à effet de l'exposition aux particules fines (PM2,5) sur la morbidité et la mortalité (Laurian, 2008; Deguen et Zmirou-Navier, 2010). Les gaz d'échappement des moteurs diesel ont été classés parmi les cancérogènes certains pour les humains par le Centre international de recherche sur le cancer (Benbrahim-Tallaa et al., 2012). Pour autant, la pollution ne touche pas uniformément le territoire et impacte différemment les populations qui y vivent (Pascal, 2009). Des inégalités d'exposition à la pollution atmosphérique existent: les personnes des catégories socioprofessionnelles les plus défavorisées peuvent être plus exposées que les autres (parce qu'elles vivent à proximité d'axes routiers très fréquentés) et elles sont plus vulnérables, car leur état de santé est plus fragile et leur accès aux soins moins bon (moins de généralistes dans certaines zones comme la Seine-Saint-Denis, évitement des frais médicaux), malgré le système de sécurité sociale français (Havard et al., 2009). Elles ont en outre moins de ressources pour éviter cette pollution ${ }^{1}$. La pollution atmosphérique peut donc être la cause d'impacts différenciés à la fois socialement et spatialement (concentration sur certaines zones habitées par des populations à faibles capitaux socioéconomiques) et ainsi s'avérer source d'inégalités environnementales.

Malgré cette percolation du thème de la santé environnementale dans les univers de la recherche, de l'opinion publique et des décideurs, de multiples débats animent la communauté de chercheurs en France sur ce que sont réellement ces inégalités environnementales. Les mettre en exergue ne pose-t-il pas d'emblée un problème méthodologique? Sont-elles une nouvelle dimension des inégalités sociospatiales ou ont-elles une acception propre? N'assigne-t-on pas de prime abord un jugement de valeur à des disparités spatiales somme toute «naturelles» dans l'allocation des biens et des maux environnementaux? Cette terminologie n'est-elle pas accusatrice avant d'être analytique? N’induit-elle pas un «impératif moral imposant [la] réduction des inégalités environnementales» (Charles, $2007: 54$ ) et obligeant donc à une action des pouvoirs publics? De toute évidence, le prisme «racial», tel qu'il a prévalu aux États-Unis au départ, est délaissé en France, et le concept de justice environnementale, ré-approprié au contexte et à la pensée hexagonale. L'expression «inégalités environnementales» se

1 Outre les choix résidentiels contraints, elles ne passent pas ou très peu de leurs week-ends en dehors de leur lieu d'habitation. 
prête en fait difficilement à une définition générique qui transcenderait les différences disciplinaires et nationales. Il est pourtant important de pouvoir s'appuyer sur une grille d'analyse nous permettant de valider ou d'invalider le concept au regard de la situation locale. La typologie des inégalités environnementales élaborée par Walker et ses collaborateurs en 2003 distingue:

- l'exposition à un impact environnemental (distribution des coûts environnementaux) comme la pollution de l'air, le risque d'inondations, etc.;

- l'accès aux ressources/aménités environnementales (distribution des bénéfices et aménités environnementales);

- la capacité d’influencer les décisions affectant l'environnement: implication de la communauté/du citoyen dans le processus de prise de décision, éducation et pédagogie, transparence;

- la justice à l'égard de l'environnement (distribution des coûts et des bénéfices entre les usagers anthropiques et écologiques).

Cette typologie semble pertinente, mais elle passe sous silence une forme d'inégalités environnementales: les effets différenciés et potentiellement sources d'inégalités des politiques environnementales en fonction des populations auxquelles celles-ci s'adressent et s'imposent. En outre, elle tait ce qui est lié aux représentations; or, la justice n'est pas un donné abstrait et général, mais une construction sociale qui se nourrit de valeurs, de perceptions collectives et individuelles du juste dans une époque et un contexte donnés (Walser, 1983; Dobson, 1998). C'est pourquoi, au-delà de la distinction usuelle entre justice distributive (qui met l'accent sur la répartition des biens et des maux) et justice procédurale (qui a trait à la procédure, à la distribution du pouvoir et à la participation à la prise de décision), il est nécessaire de s’appuyer sur une taxinomie plus fine des enjeux qui intègre la reconnaissance de la diversité des populations, mais aussi les représentations de ces populations à l'égard de leur environnement (Schlosberg, 2002; Fraser, 2005).

L'émergence de la thématique des inégalités environnementales et leur prise en compte dans les discours des acteurs et dans l'action collective demandent donc de comprendre ce qui apparaît juste ou injuste dans un cadre précis et qui peut justifier une place sur l'agenda dans l'action publique. Cette thématique exige également de voir comment la question environnementale est saisie par les populations, les chercheurs et autres acteurs pour être mise en regard de la question sociale (Chardonnel et al., 2012).

Nous avons voulu explorer ces aspects. Dans l'étude réalisée ex ante (2011-2012) à la mise en place d'un dispositif de réduction de la circulation automobile dans les grandes agglomérations françaises (et particulièrement dans celle de Plaine Commune), nous avons cherché à savoir si la mesure allait renforcer les inégalités sociales et territoriales et créer des inégalités environnementales ou, au contraire, si elle allait fondamentalement améliorer le bien commun et, par ricochet, le bien-être et la santé des plus démunis. De fait, l'hypothèse première de la recherche était qu'une interdiction de circulation des vieux véhicules allait d'abord toucher les plus pauvres, davantage équipés de vieilles autos ou motos, et moins capables de les renouveler en raison d'un budget contraint (Orfeuil, 2004; Fol, 2009). Une telle mesure allait donc accentuer leurs difficultés à se déplacer et à pouvoir atteindre certaines fonctions urbaines (services, emplois) (Wenglenski, 2006). Plus encore, la mesure imputait tacitement la responsabilité de 
la pollution aux véhicules anciens et faisait supporter les coûts aux moins aisés. Or, la littérature concernant l'internalisation des coûts générés par le trafic automobile (urban road pricing) montre combien les enjeux d'équité influent sur l'acceptabilité et la difficulté à traiter d'un problème sanitaire et environnemental dans des termes qui sont subis comme punitifs et injustes (Viegas, 2001; Eliasson et Mattsson, 2006).

Dans le même temps, les populations pauvres semblent les plus exposées à la pollution atmosphérique $^{2}$; la distribution géographique des populations selon les revenus tend à montrer que les moins fortunés subissent de manière plus forte la pollution, notamment parce qu'ils sont plus susceptibles d'habiter près des infrastructures, des axes routiers, etc., non par choix, mais par opportunité immobilière (Gueymard, 2009). Leurs choix résidentiels sont en effet plus contraints que ceux des ménages ayant des ressources financières élevées.

Dans cet article, nous rappellerons d'abord les grandes lignes de la mesure ainsi que le contexte français dans lequel celle-ci était censée s'appliquer ${ }^{3}$. Nous préciserons également les caractéristiques du terrain d'étude. Dans un second temps, nous mettrons en avant les résultats recueillis grâce à des entretiens semi-directifs et à une enquêtequestionnaire, à la fois sur les plans subjectif (ressenti des populations à l'égard de la possible application de cette mesure) et objectif (quelles répercussions et possibilité d'adaptation au regard des «capitaux de mobilité» [Kaufmann, 2004] de l'individu).

Cela nous permettra de considérer que l'inégalité environnementale ne se lit pas nécessairement comme la superposition d'un «mal environnemental» (exposition à une pollution, une nuisance ou un risque naturel ou industriel) aux caractéristiques sociales d'une population habitant sur un territoire donné. Cette lecture exige une approche géographique, sociale et psychologique plus subtile pour comprendre comment des situations ou des mesures liées à l'environnement peuvent être appréhendées comme injustes, et quels mécanismes accroissent le poids des maux socioéconomiques de certaines populations.

\section{La mise en place des zones d'actions prioritaires: éléments de contexte}

La Directive européenne 2008/50/CE sur la qualité de l'air fixe des valeurs maximales d'exposition de la population aux polluants PM10 et PM2,5 (particules fines en suspension dans l'air) et aux oxydes d'azote $\left(\mathrm{NO}_{2}, \mathrm{NO}_{3}\right)$. Les valeurs limites de présence de polluants atmosphériques fixées par la Commission européenne ne sont pas respectées en France dans 16 zones, et singulièrement en Île-de-France.

Plaine Commune, intercommunalité réunissant huit communes, est particulièrement concernée (figure 1), d'une part, parce que les villes de Saint-Denis et d'Aubervilliers sont limitrophes du boulevard périphérique, générateur de pollution; d’autre part, parce que le territoire de l'agglomération est sillonné de grands axes très passants (la A86, la A1, l'ex-RN1, la RN2 et les autres radiales) et qu'il est par ailleurs bordé par la A6.

2 De nombreuses études cherchent à corroborer le lien entre exposition à la pollution atmosphérique et populations socioéconomiquement défavorisées (Stroh et al., 2005; Laurent et Bard, 2007). Ce lien doit évidemment être mis en perspective avec le territoire étudié.

3 Le projet a été finalement enterré lors de l'arrivée d'un nouveau gouvernement. Cependant des mesures approchantes sont actuellement en cours d'examen par la mairie de Paris. 


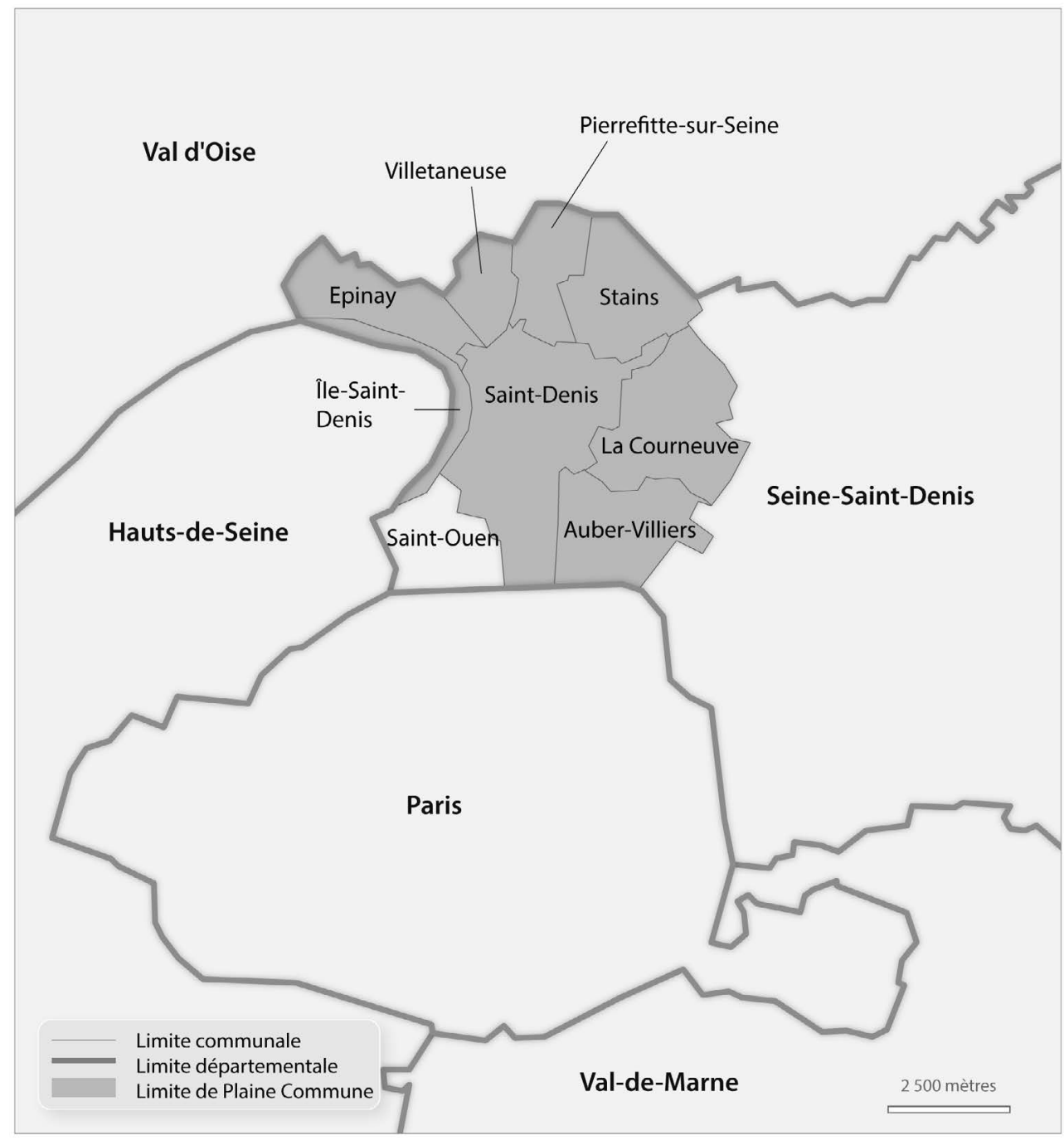

\section{L'imposition des zones d'actions prioritaries comme outil d'action publique}

En l'absence d'action urgente significative concernant la pollution atmosphérique, la France s'exposait devant la Cour de justice européenne à une amende de plusieurs millions d'euros ainsi qu'à des astreintes journalières élevées. Les pouvoirs publics étaient donc dans l'obligation d'intervenir et de bien identifier les objectifs qu'ils souhaitaient atteindre. Les émissions de particules sont principalement liées au trafic automobile et au chauffage au bois dans le secteur résidentiel. La pollution liée aux oxydes d'azote est, quant à elle, principalement liée au trafic routier. La loi Grenelle I avait déjà lancé un «Plan particules». La loi Grenelle II a mis à disposition des communes ou groupements de communes de plus de 100000 habitants un outil: 
la zone d'actions prioritaires pour l'air (ZAPA). Celle-ci est directement inspirée des Low Emission Zones qui ont été expérimentées dans plusieurs grandes villes européennes (Londres, Berlin, Milan...) (Wolff et Perry, 2010). Il s'agit d'interdire la circulation des véhicules anciens, en prenant en compte les normes Euro et le type de motorisation des véhicules (le diesel étant plus producteur de particules que l'essence ${ }^{4}$. . L'Agence de l'environnement et de la maîtrise de l'énergie a donc lancé, en novembre 2010, un appel à projets auprès d'agglomérations volontaires souhaitant expérimenter ce nouveau dispositif sur leur territoire. Paris et Plaine Commune se sont portées candidates et ont décidé de joindre leurs efforts pour monter ce dossier.

\section{Méthodologie}

Des études communes à partir de différents scénarios ont été menées sur les deux zones, notamment par l'Association de surveillance de la qualité de l'air en Île-deFrance (AIRPARIF) et la Direction régionale et interdépartementale de l'Équipement et de l'Aménagement Île-de-France, service déconcentré de l'État (DRIEA) (réflexion sur le périmètre le plus efficace en termes de pollution de l'air). Les résultats explicités ciaprès proviennent de l'étude sociologique commanditée par Plaine Commune. L'étude a été effectuée sur la base d'une méthodologie couplant le quantitatif (enquête auprès de trois populations - représentants d'entreprises, salariés et habitants - menée par questionnaire Internet et par téléphone ${ }^{5}$ ) et le qualitatif (entretiens semi-directifs ${ }^{6}$ qui, pour les habitants, se couplaient de la proposition de tenir un journal de bord de leurs déplacements pendant une semaine) (tableau 1). L’objectif de ce dispositif méthodologique était de confronter la parole à l'acte transcrit dans un carnet et d'avoir une meilleure appréhension de l'amplitude des déplacements quotidiens des personnes.

\section{Tableau 1 Présentation de la méthodologie de l'enquête}

\begin{tabular}{lll}
\hline & \multicolumn{1}{c}{ Volet qualitatif } & Volet quantitatif \\
\hline Entreprises & 19 entretiens semi-directifs approfondis & 418 questionnaires Internet \\
\hline Salariés & 15 entretiens semi-directifs approfondis & 1202 questionnaires Internet \\
\hline Habitants & $\begin{array}{l}18 \text { entretiens semi-directifs approfondis }+ \\
10 \text { suivis de journal de bord }\end{array}$ & $\begin{array}{l}419 \text { questionnaires par } \\
\text { téléphone et de visu }\end{array}$ \\
\hline
\end{tabular}

4 Pendant longtemps, les pouvoirs publics ont incité à l'achat de véhicules diesel car diffusant moins de $\mathrm{CO}_{2}$ et donc participant moins au réchauffement climatique, mais d’autres effets ont été minimisés. D'où la difficulté aujourd'hui de communiquer sur des mesures et des objectifs en contradiction avec ce qui a été dit plus tôt: ce type de «retournement» nourrit la méfiance envers les institutions et l'expertise.

5 Choix aléatoire des répondants sur l'ensemble des huit communes de Plaine Commune à partir de l'annuaire téléphonique et des listes d'entreprises.

6 La question finale du questionnaire visait à savoir si la personne interrogée était prête à se soumettre à un entretien plus complet. La prise de rendez-vous s'effectuait ensuite par un enquêteur qui devait vérifier la diversité des profils et la bonne distribution dans l'ensemble de l'intercommunalité des personnes ainsi interrogées. 
Les dimensions abordées dans le questionnaire et le guide d'entretien pour les salariés et habitants concernaient la description de la mobilité personnelle des individus, ainsi que la description de leurs déplacements dans le cadre de leur activité professionnelle? Étaient ensuite abordées plus précisément la mobilité motorisée individuelle (usages et représentations) et la part de la mobilité «douce» dans les déplacements quotidiens (marche à pied, tous les modes de transport, desserte, etc.). Le dernier volet se focalisait sur la perception de l'environnement, sur l'impact de la pollution de l'air sur la santé, avant d'expliquer la potentielle application d'une limitation de circulation des véhicules sur un périmètre donné. À partir de-là, on demandait aux individus leur sentiment sur la mesure, leur degré de «concernement» (Brunet, 2008), ainsi que la manière dont ils pensaient pouvoir s’adapter.

Des entretiens avec des personnes-ressources (dont un représentant de la chambre de commerce et d'industrie de Seine-Saint-Denis, un médecin épidémiologiste, un représentant de l'autorité organisatrice des transports en commun en Île-de-France, un représentant de l'opérateur principal des transports urbains sur le secteur de Plaine Commune, etc.) ont également eu lieu pour connaître les positionnements institutionnels, les compromis à réaliser pour mettre en place une restriction de la circulation et déterminer le rôle possible de l'expertise dans la construction de la mesure.

\section{Des droits-créances contradictoires?}

Le dispositif ZAPA n'intervenait pas dans un désert législatif ou réglementaire; il prolongeait des actions nationales et locales menées depuis plusieurs années sur la question de la pollution. Pourtant, cette prise en compte publique des conséquences sanitaires néfastes de l'exposition aux émissions de particules est relativement récente, au regard des textes faisant la promotion de la mobilité. La loi sur les transports intérieurs de 1982 a ainsi affirmé le droit au transport, droit consolidé par la loi de lutte contre les exclusions, en 1988, et la loi Solidarité et renouvellement urbain de 2001 (art. 123). En fait, peu à peu, les dispositions législatives ont pris en compte les inégalités d'accès, plus seulement du point de vue de la disponibilité d'infrastructures, mais de celui de la mobilité individuelle (Gallez, 2010). Ce n'est que bien plus tard qu'un «droit à chacun à respirer un air qui ne nuise pas à sa santé» est apparu. Dans les années 1990, des textes législatifs ont institué les prémisses d'une réflexion sur la mobilité durable: la Loi sur l'air et l'utilisation rationnelle de l'énergie (LAURE) de 1996 qui «cherche à apporter une réponse territoriale en généralisant la surveillance de la qualité de l'air et en développant des stratégies planificatrices à caractère préventif» (Charles, 2009). Elle a par ailleurs mis en avant la question de la santé, ce qui était innovant.

La mise en place des ZAPA semblait venir rompre un équilibre en affichant délibérément une volonté de limiter l'émission de particules sans s'occuper de mobilité. Le droit à un environnement sain semblait être dissocié du droit à la mobilité; deux "droits» qui ont émergé récemment et qui semblaient être devenus des droits-créances. De fait, à partir du XIX ${ }^{\mathrm{e}}$ siècle, sont apparus un ensemble de droits collectifs, reconnus à une personne juridique plus importante que l'individu lui-même. Le préambule de la constitution du 27 octobre 1946, en France, a établi un ensemble de droits-créances (dont le droit au travail), qui impliquent une action effective de l'État et un engagement

7 Le questionnaire pour les entreprises était plus court et se focalisait surtout sur les contraintes économiques liées au besoin de transport. 
de moyens, financiers notamment, pour y accéder. La société civile n’a cessé de tenter d'élargir ces droits-créances et, d'ailleurs, certains engagements de l'État sur la question environnementale peuvent faire figure de droits collectifs, quasi droits-créances.

\section{Les caractéristiques sociospatiales et environnementales de Plaine Commune}

Plaine Commune est une agglomération densément peuplée de 346209 habitants. Le pourcentage de ménages y résidant et vivant sous le seuil de pauvreté atteint $31 \%$, et 20,6\% des habitants sont au chômage contre 17,2\% en Seine-Saint-Denis et 11,6\% en Île-de-France (département et région où se situe Plaine Commune) (INSEE, 2009). Le PIB moyen de l'agglomération est inférieur de moitié à celui de Paris. En termes de niveau de revenu de ses habitants, Plaine Commune est la 154e agglomération sur 155. La mise en perspective du salaire net moyen horaire, en 2010, montre une différence entre Plaine Commune $(11,4 €)$ et le reste de la France $(13,4 €)$.

Dans le même temps, le territoire de l'agglomération est particulièrement exposé à la pollution atmosphérique (94 jours de dépassement des seuils d'exposition à la pollution aux particules sur la station de l'autoroute A1 depuis le 1er janvier 2012) et à certaines nuisances (bruit le long des grands axes routiers, bruit des avions, notamment sur la ville de Stains au nord de l'intercommunalité).

En outre, l'ensemble du territoire ne bénéficie pas de la même couverture en transport en commun même si, au moment où l'étude était effectuée, des travaux d'aménagement de nouvelles lignes de tramway (ou la prolongation de lignes existantes) devaient prochainement mieux mailler les villes du nord. Ces dernières (Epinay, Stains), moins proches de Paris, s'avèrent moins bien desservies et seulement de manière radiale, non transversale, par le RER. Celles du sud (Saint-Denis, Aubervilliers) cumulent au contraire les moyens de transport lourd (métro, RER, tramway) et léger (bus). La Courneuve reste cependant surtout maillée dans ses pourtours.

Le taux de motorisation est, pour l'ensemble de l'agglomération, de 58\% environ, avec des variations importantes entre Aubervilliers (48\%) et des villes comme Villetaneuse (64\%) ou Pierrefitte (66\%). Il faut rappeler qu'outre l'impossibilité financière d'acquérir une voiture et de l'entretenir, sa possession par les personnes ou ménages à faible revenu peut être source de nombreuses contraintes (Chevallier, 2002) : véhicule ancien, donc souvent en panne; difficulté à respecter les normes environnementales, à payer les assurances, d'où, quand elles ne sont pas acquittées, des déplacements de faible amplitude limitant le risque d'être «pris». Par ailleurs, les propriétaires de ces véhicules ont une consommation en carburant très rationnalisée, pour en restreindre le poids sur leur budget. Les pauvres se déplacent moins que les autres ménages et réalisent des trajets plus courts. À ce titre, ils polluent moins en masse que d'autres ménages. Leur assigner la charge de la responsabilité environnementale pose donc problème, surtout si ne sont pas réfléchies des mesures d'accompagnement spécifiques.

Toutefois, les analyses des politiques publiques en matière d'amélioration de la qualité de l'air, par l'action sur les transports (instauration de péages urbains par exemple), donnent des résultats ambigus. Certains auteurs (Evans, 1992; Small, 1992) considèrent que les voyageurs aux revenus les plus faibles qui, en règle générale, se déplacent en transport en commun ou à pied, seraient finalement bénéficiaires de 
l'application des mesures de type ZAPA. Les avantages seraient pour eux l'amélioration de la vitesse des transports collectifs grâce à une meilleure fluidité du trafic, ainsi que l'affectation partielle des recettes tirées de la politique ZAPA aux transports collectifs et aux piétons. Qu'en est-il pour Plaine Commune?

\title{
Une mesure d'interdiction de la circulation des voitures anciennes jugée injuste et injustifiée
}

Il était intéressant de confronter la connaissance qu'ont les individus, avec leur ressenti, de la pollution atmosphérique et de ses conséquences par rapport à l'instauration d'une mesure d'interdiction de la pollution. Cela permettait de voir si les aspects sanitaires d'une mesure étaient bien évalués et pouvaient justifier de possibles conséquences sociales inégales. Or, il est apparu que la pollution de l'air n'était pas une préoccupation majeure et que l'instauration d'une ZAPA était moins appréhendée du point de vue de sa possible efficacité environnementale que de ses conséquences économiques directes sur les ménages. Ce que d'autres études avaient déjà mis en exergue:

\begin{abstract}
Although the LEZ would also put the polluter-pays principle into operation, the group opposed it, because it was not seen to serve a valid purpose. The important conclusion that follows is that the question of allocating the financial burden of a policy in the public mind is not independent of how it perceives the same policy's outcomes: perceptions of environmental equity are not independent of economic equity. (Dietz et Atkinson, 2005 : 453)
\end{abstract}

\section{Une moindre conscientisation sur les questions de pollution atmosphérique}

Nombreux sont les habitants interrogés qui considèrent Plaine Commune comme polluée (63\%). Cependant, cette statistique n'illustre pas le degré de connaissance en la matière, mais plutôt un sentiment général.

Contrairement à la pollution sonore ou visuelle, la pollution atmosphérique est quasiment invisible et, par là-même, souvent mal appréhendée. Les entretiens approfondis illustrent abondamment le fait que les interviewés ont du mal à se représenter de quoi il s'agit. Ils en ont souvent entendu parler, savent que la région parisienne est particulièrement touchée mais, pour eux, cette pollution n'a pas de matérialité intrinsèque.

Le problème dont les pouvoirs publics se sont emparés n’a pas de véritable écho dans la société civile. Le terrain de Plaine Commune illustre qu'il n'existe pas réellement de «conscience collective [et partagée] au quotidien [qui] passe par des capteurs et les messages vulgarisés de la science mais aussi par la construction active de théories profanes du risque» (Morin, 2006: 169).

Toutefois, de manière partielle, au travers de certains vecteurs, on peut déceler chez les habitants les prémisses d'une réflexion au sujet de la pollution. Ainsi, les interviewés semblent-ils mieux identifier la problématique du réchauffement climatique et de l'effet des gaz à effet de serre, car elle est plus médiatisée. 
Par ailleurs, les différents entretiens montrent qu'il n'existe pas de consensus pour établir des liens de cause à effet et désigner le ou les principaux contributeurs à la pollution atmosphérique. La circulation automobile est souvent évoquée comme cause de la pollution, mais la part de la voiture individuelle est souvent amoindrie, car les personnes interrogées ne souhaitent pas remettre en cause leurs propres pratiques. Par conséquent, pour elles, ce serait avant tout les camions et poids lourds qui pollueraient, tout comme les bus. Dans les discours, la volonté de consentir à des efforts personnels pour diminuer la pollution atmosphérique apparaît donc rarement.

Cette conscientisation balbutiante et cette représentation floue de la responsabilité constituent des obstacles à la reconnaissance du bien fondé des mesures coercitives ou économiques visant à limiter l'usage de l'automobile. L'utilisation du principe pollueur-payeur semble difficile à faire admettre en ces termes, puisqu'il existe une disjonction entre les représentations des autorités et celles des populations concernant le pollueur. D’ailleurs, en règle générale, la désignation d’un coupable (idéal) rend souvent plus aisées les mobilisations citoyennes et l'acceptation de telles mesures; ce qui n'est pas le cas ici, ainsi que l'explique, par exemple, le médecin épidémiologiste de l'École des Hautes Études en Santé Publique (EHESP) que nous avons rencontré:

Je connais les associations travaillant sur la santé et l'environnement. Elles se mobilisent que lorsqu' «un gros méchant» est identifiable. Or, pour la pollution atmosphérique il n’y en a pas, ou c'est monsieur tout le monde. [...]. Les gens ne voient pas la pollution, ne la sentent pas, sauf quand ils souffrent de certaines pathologies, ils ne cherchent pas de coupables, dès lors que ce n'est pas une usine bien identifiée, un entrepôt de camions qui génèrent beaucoup de nuisances et de pollution.

Ces premiers résultats mettent à mal le registre de justification des pouvoirs publics, et laissent à penser que la mesure ne sera pas facilement acceptée, car la population n’adhère pas aux raisons avancées.

\section{Un sentiment profond d'injustice}

Quand, après avoir interrogé les habitants de Plaine Commune sur leur mobilité quotidienne et sur leurs représentations de l'environnement et de la pollution de l'air, nous leur demandons leur opinion sur la mise en place d'une ZAPA, nous notons un manque d'enthousiasme, toutes catégories confondues. Si, sur le principe, les habitants sont à une faible majorité d'accord avec un tel projet en raison de ses ambitions sanitaires (52\%), ils manifestent quasi immédiatement une sensation d'injustice.

From a psychological point of view perceived justice, among others, is of major concern as a basic requirement for acceptability. Justice, as people perceive it, may differ from objective distribution of costs and benefits but surely depends on it as one major parameter influencing personal perceptions. And, as with most personally mediated perceptions, it differs not only between different situations (intraindividual variance) but also between people in the same situation and even between people with comparable objective costs and benefits (interindividual variance) (Schade et Schlage, $2003: 49-50$ ).

Les jugements et les sentiments de justice des individus sont complexes (Boudon, 1995; Dubet, 2005), car irréductibles à des théories ou à des procédures abstraites de répartition des biens (proportionnalité, rétribution au mérite, etc.). Les particuliers pensent le juste dans un contexte social particulier (systèmes d'interaction) et un cadre cognitif concret (Kellerhals et Modak, 1997). À ce titre, les individus se réfèrent 
à des situations existantes pour juger d'un projet non encore appliqué et prennent en compte, pour élaborer leur jugement, plusieurs facteurs (rarement de manière simultanée, mais plutôt de manière exclusive) :

- l'origine de l'objet: en l'occurrence, il s'agit de la personne ou de l'institution qui prend la décision (l’État et les collectivités territoriales, ici);

- les raisons qui guident l'action (quels objectifs sont poursuivis? sont-ils légitimes?) ;

- l'objet lui-même, sa nature: la mise en place d'une ZAPA est une mesure réglementaire top-down appliquée par les collectivités locales. Ce type d'outil peut susciter en lui-même des interrogations par rapport à d'autres modes d'action publique (incitations économiques, partenariat avec les parties prenantes d'un sujet) ;

- les conséquences supposées de la mesure, c'est-à-dire sa dimension distributive;

- la procédure adoptée pour mettre l'objet en place (dimension procédurale).

Ces différents angles d'appréhension de l'objet ZAPA permettent de mieux comprendre où se niche le sentiment d'injustice. C'est d'abord par les conséquences supputées de la mesure que les individus réagissent: ils considèrent que les publics cibles sont les ménages pauvres et cela apparaît comme inacceptable, d'autant plus que, comme nous l'avons vu, la légitimité de l'action sur la pollution de l'air est à construire. Pour les gens, cette pollution n'est pas une préoccupation sanitaire majeure. Aussi, les individus déportent-ils les raisons qui guident l'action vers d'autres impératifs "cachés» des pouvoirs publics. En effet, le sentiment d'injustice se cristallise également dans l'identification présumée des bénéficiaires : l'industrie automobile. À ce titre, c'est la légitimité de la mesure qui est remise en cause parce que les explications avancées par les pouvoirs publics (problème de santé publique, lutte contre la pollution, etc.) occulteraient sciemment des raisons économiques. La figure 2 présente ainsi, à la fois, le schéma de justification fourni par les pouvoirs publics et les représentations qui y sont associées par les individus (en grisé).

Plus encore, derrière cette critique, peut se dissimuler un reproche beaucoup plus large à l'égard des mesures environnementales, selon lequel se préoccuper d'environnement et de développement durable est toujours onéreux pour certains (les "petites gens») et avantageux pour d'autres (certaines entreprises). Cette présomption participe d'une méfiance institutionnelle à l'égard de toute rhétorique environnementale.

Que les ménages modestes ne soient pas systématiquement les payeurs ou les laissés-pour-compte constitue l'une des préoccupations principales. Par conséquent, une grande partie de la population partage une vision «rawlsienne» de la justice: ils acceptent l'amélioration globale de la situation sanitaire, mais la mesure y menant ne doit pas se faire au désavantage des ménages pauvres. D’où la demande quasi unanime d'aides ciblées aux ménages en difficulté pour renouveler leur automobile.

Du point de vue des répondants, l'injustice semble aussi caractérisée, dans la mesure où ils considèrent qu'on fait payer aux citoyens les erreurs des urbanistes, des aménageurs et des décideurs publics, qui n’ont pas su bien coordonner développement économique, poussée démographique, urbanisme et transports. Certaines personnes 
ayant participé à l'enquête soulignent que les bassins d'emploi ne sont pas en concordance avec les bassins de vie et les compétences déployées sur un territoire. Elles se demandent pourquoi les pouvoirs publics ne cherchent pas avant tout à corriger cette disjonction et à avoir une approche plus globale de la planification des villes, mêlant le transport, le développement économique, l'emploi, etc. (Ecola et Light, 2010).

\section{Figure 2 Acteurs et représentations de la politique publique de diminution des particules par l'instauration d'une ZAPA}

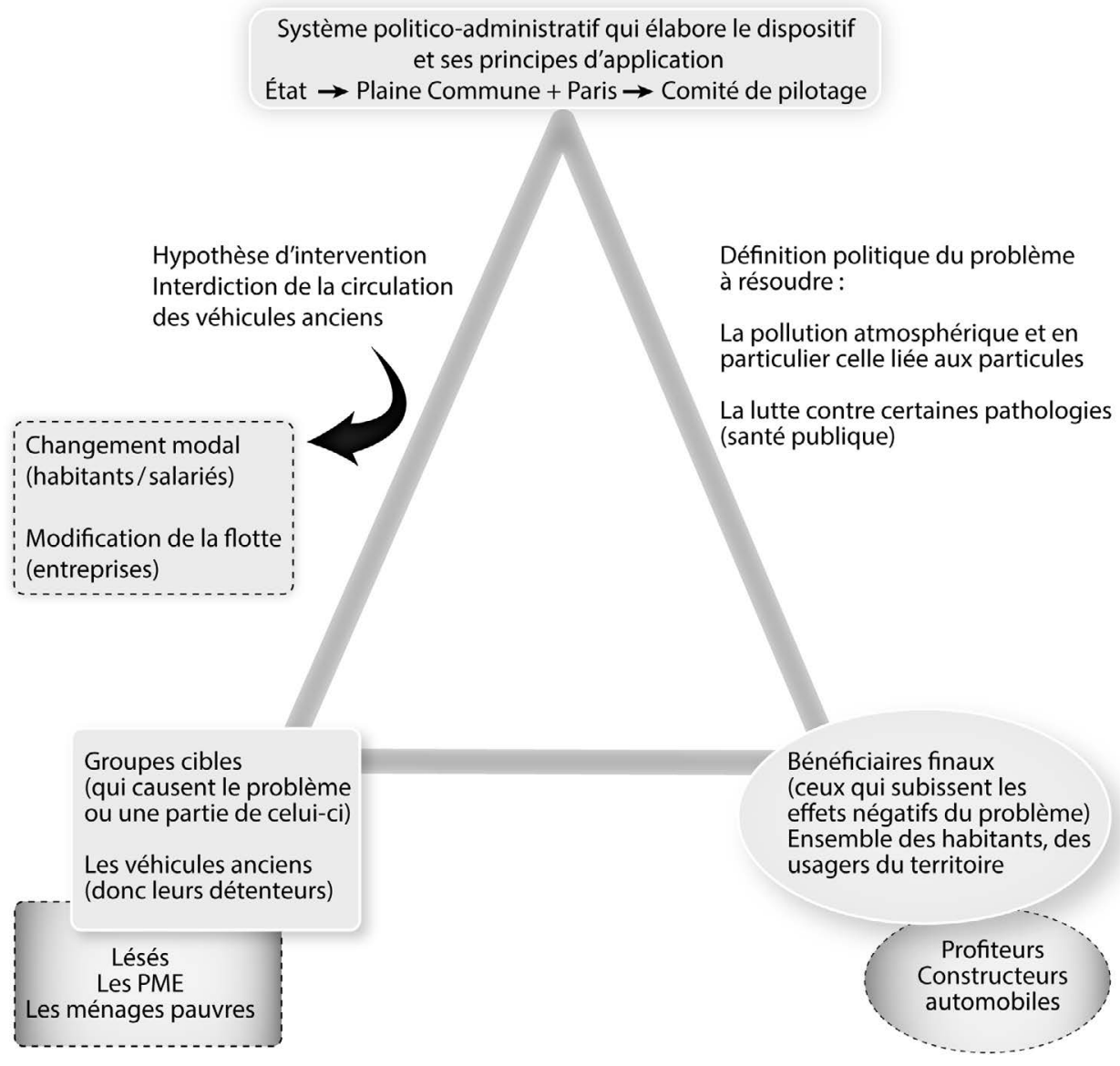

Source : Knoepfet et al. 2001

Ces réponses donnent à voir que les fondements de la mesure ne sont pas acceptés, d'une part parce que, dans les faits, les plus pauvres seront vraisemblablement les plus impactés, mais également parce qu'on leur impute une pollution à laquelle ils ne contribuent pas plus que les autres, selon les représentations les plus communément partagées. D’ailleurs, l'enquête nationale transport et déplacements 2008 démontre que plus les revenus d'un ménage sont élevés, plus les personnes se déplacent en voiture (CGDD, 2010: 47). 
Il est intéressant de noter qu'une étude avait également été menée en amont de la mise en place d'une ZAPA dans l'agglomération grenobloise et qu'elle donne des résultats approchants (La Branche et Charles, 2012).

Au-delà de ce registre se focalisant sur les perceptions, nous avons cherché à voir ce qu'il en est objectivement, en travaillant sur les capacités d'adaptation des individus. Analyser les résultats de l'enquête de ce point de vue oblige à revoir ce qui semble «injuste» au premier abord, car les capacités et modalités d'adaptation montrent qu'on ne peut s'arrêter à une classification par catégorie de revenus.

\section{Une capacité d'adaptation divergente des populations}

Si le sentiment d'iniquité obère l'acceptabilité d'une telle mesure, ainsi qu'ont pu également le montrer d'autres études (Schade et Schlag, 2003), les individus, même les plus démunis, ne sont pas dénués de ressources pour faire face à la mesure. Pour le montrer, au-delà des discours et des représentations des habitants à l'égard de la mesure, nous avons donc analysé les réponses concernant leur mobilité quotidienne afin de comprendre quelle était leur capacité de s'adapter. Nous avons cherché à savoir si la mesure allait constituer un handicap pour leurs déplacements et si les réponses à ces questions étaient fonction de certaines variables sociodémographiques (âge, revenu, etc.) ou spatiales (lieu d'habitation, etc.). Allait-on réellement créer, avec cette mesure, des situations d'inégalité ou renforcer celles existantes, mais non traitées? Cette seconde phase permettait de confronter une représentation subjective des conséquences aux capacités objectivées des individus d'ajuster leurs habitudes.

Travailler sur la capacité d'adaptation nous a demandé, sous l'inspiration de la thèse d'Anaïs Rocci (2007) ${ }^{8}$, d'isoler les variables les plus pertinentes au regard des entretiens et des questionnaires. Celles-ci se sont avérées être:

- le type d'usager au regard de «son portefeuille personnel de droits d'accès» (compétences et acquis en termes de mobilité: permis, savoir faire du vélo, etc., que nous avions pu définir lors de la première partie de notre questionnaire) (Kauffmann et al., 2001 ; Flamm, 2004) ;

- le rapport aux véhicules (rapport affectif ou strictement utilitaire à l'égard du transport motorisé individuel) ;

- le rapport aux autres modes de transport (rejet du transport en commun, fréquentation discontinue ou journalière), pour considérer finalement les potentialités de changements de mode. Ces derniers sont implicitement visés puisqu'ils permettraient concrètement de diminuer les particules et d'améliorer la santé publique. Les pouvoirs publics avaient cependant deux incertitudes liées à la mise en place du projet de ZAPA: la part de la population prête à renouveler son véhicule en raison de la dépendance forte à la mobilité motorisée (Dupuy, 2006) et celle préférant se reporter sur le transport en commun.

8 L'auteure a notamment mis en lien le rapport aux modes de transport (affectif, instrumental) avec la potentialité de changement de modes, et distingué trois types d'usagers au départ: les automobilistes exclusifs, les multimodaux et les alternatifs. 
Pourquoi ne pas retenir d'autres variables explicatives? Il est apparu que le lieu d'habitation n'était pas significatif dans les réponses données, bien que le scénario testé (périmètre d'interdiction comprenant l'ensemble des villes situées à «l'intérieur» de l'A86, comme le présente la figure 3) couvre seulement une partie des villes de l'agglomération (celles du sud de Plaine Commune).

\section{Figure 3 Schéma du périmètre de limitation de la circulation des véhicules anciens}

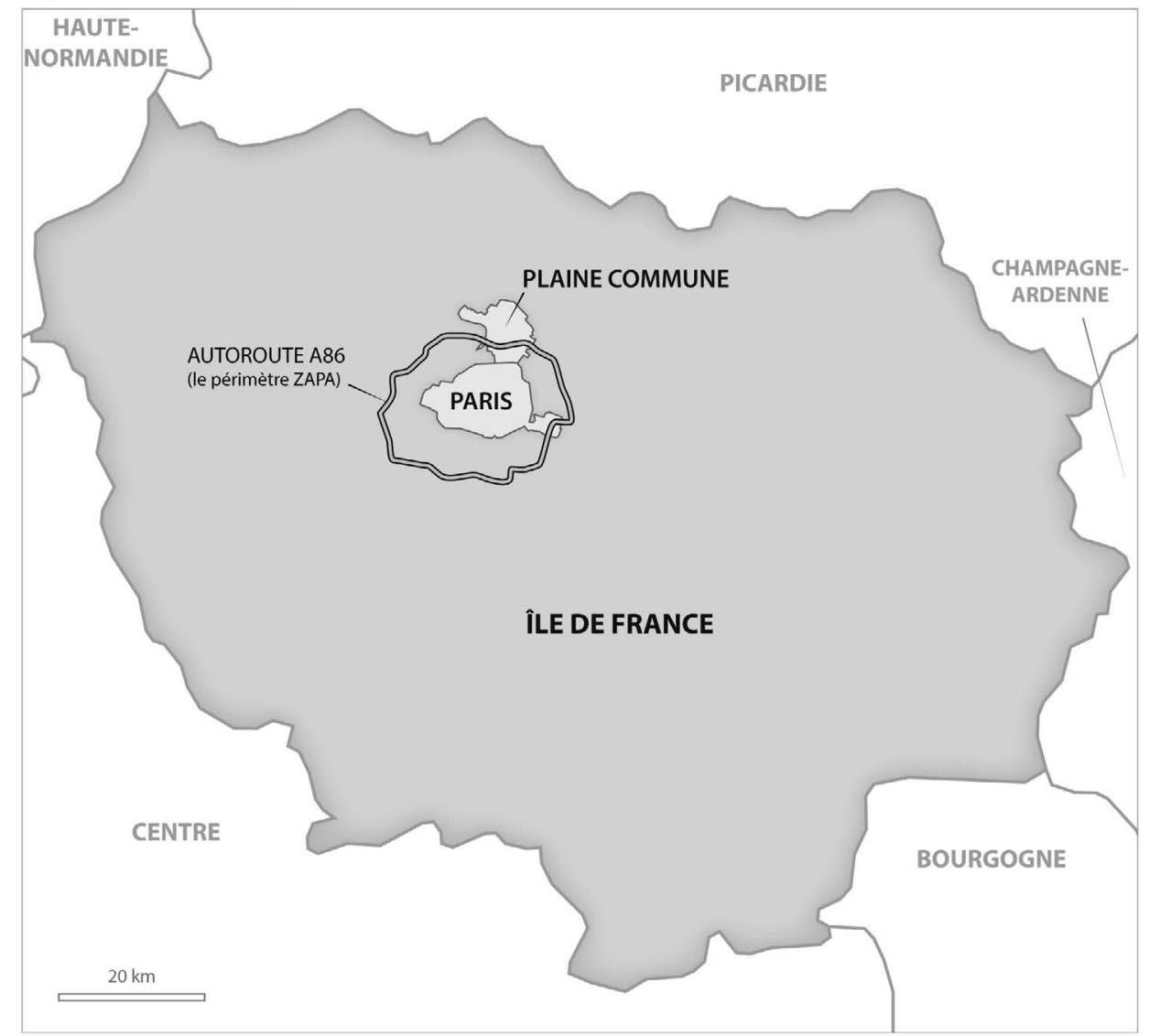

Auteur : Julie Gobert

De même, contrairement à ce que nous aurions pu croire dans un premier temps et qui correspondrait à un phénomène de renforcement des inégalités sociales, la variable revenu n'est pas déterminante de manière univoque. Ce que privilégient les individus concernés par la disposition (c'est-à-dire ceux qui possèdent un véhicule ne pouvant plus circuler) est le changement de mode de transport, surtout pour les populations précaires (35\% des ménages percevant moins de $1500 €$ préfèrent cette solution, 30\% de ceux touchant plus de $5000 €$ ). Plusieurs choix leur étaient en effet proposés: changer de véhicule, changer de mode de transport, éviter le périmètre d'interdiction, installer un filtre à particules (cette modification étant évaluée financièrement pour que les individus aient une vision des coûts à consentir). 
On voit surtout s'instaurer une différence plus importante quand il s'agit du changement de véhicule (les ménages aisés concernés étaient peu nombreux, mais plus prompts à choisir cette option que les ménages à bas revenus) ou d'une comparaison entre les salariés précaires ${ }^{9}$ et les salariés des grandes entreprises (qui proposent notamment à leurs employés des plans de déplacement ou d'autres dispositifs).

De plus, nous avons constaté que la mauvaise desserte peut en partie justifier qu'un individu soit plutôt un unimodal satisfait ou un unimodal contraint (personne ne prenant qu'un type de transport: la voiture ou la moto). En revanche, la bonne desserte n'est pas décisive dans les comportements ; les individus peuvent résider sur un territoire qui compte des stations de RER, de métro, de tramway ou de bus à côté de chez eux, et tout de même préférer le véhicule motorisé individuel.

La conscience environnementale est un facteur décisif pour l'altermobile radical quand celui-ci correspond au second type (personne engagée) présenté ci-après. Elle l'est aussi plus partiellement pour le multimodal. En revanche, certains usagers unimodaux satisfaits peuvent avoir conscience de leur empreinte écologique et ne pas vouloir renoncer à leur auto-moto-mobilité, qui est synonyme de liberté, d'affranchissement des contraintes du collectif. Cette variable est donc non discriminante pour tous les profils.

Sur la base des critères qui nous sont apparus déterminants dans l'adaptabilité des individus, nous avons pu distinguer différents profils, grâce aux entretiens semi-directifs:

- l'usager multimodal (qui utilise plusieurs moyens de transport dont les véhicules motorisés individuels) dont le portefeuille de compétences de mobilité est important (voiture, marche ou vélo ou transport en commun). Son adaptabilité à une ZAPA est forte;

- l'usager unimodal (qui utilise seulement la voiture ou la moto) satisfait avec un portefeuille de compétences de mobilité restreint (essentiellement la voiture, ponctuellement d'autres modes quand c'est indispensable) dont la capacité d'adaptation à une mesure de restriction de la circulation est faible;

- l'usager unimodal contraint qui n'a pas de desserte près de chez lui ou là où il se rend. Il peut de plus subir des horaires décalés et faire face à des dépenses importantes liées au véhicule. Il s’adaptera difficilement à une ZAPA en raison de contraintes extérieures;

- le non-usager (du véhicule individuel motorisé) qui souhaite augmenter son «portefeuille des droits d'accès » afin d'accéder à l'automobilité (étudiants, ménages pauvres, etc.) ;

- l'altermobile radical (qui n’utilise pas la voiture dans ses déplacements) comprend deux sous-types: celui qui possède un portefeuille de compétences ne comprenant pas le permis, mais ne s'en trouvant pas incommodé (il s'agit particulièrement des femmes et des personnes

9 Dont la mobilité n'est pas une préoccupation pour les entreprises qui les emploient. Ces salariés précaires doivent se débrouiller pour se rendre sur leur lieu de travail à des horaires parfois décalés, en l'absence de bonne desserte et malgré des ressources faibles. 
âgées), celui qui refuse la possession d'une voiture et pratique très ponctuellement le partage d'auto ou le covoiturage (non compris dans notre échantillon).

Pour revenir à notre questionnement originel, nous nous apercevons que l'adaptation à la mesure et le report modal sont moins des questions de revenus que de compétences en mobilité (Eliasson et Mattson, 2006). A priori, ce ne sont pas les individus pauvres qui sont les moins dotés de ces compétences, car étant donné les contraintes budgétaires qu'ils subissent, ils adoptent des stratégies complexes pour lisser les coûts de transport. Ils sont donc souvent des multimodaux maîtrisant les codes des différents types de transport. Pour autant, ils sont ceux pour qui la mesure aurait le plus d'incidence économique à court terme, car ils ne sont pas en capacité de renouveler leur véhicule.

En outre, les salariés dont les horaires sont décalés ou exerçant dans des zones d'activités non maillées par le transport en commun se retrouveraient pénalisés. Or, ce sont souvent les plus pauvres. Pour eux, la voiture est quasiment «un outil de travail» dont ils ne peuvent se passer. Les salariés précaires, déjà très adaptables et très flexibles, s'adapteront à une ZAPA comme ils le font continuellement, mais ce sera au prix d'une diminution de leur bien-être global.

\begin{abstract}
J'arrive à 4h/4h15 [au travail] et je repars à...il y a des jours où il y a plus de travail, il m'arrive de partir à $11 \mathrm{~h} 15$; et d'autres jours il y a moins de travail, et je pars à 10h, parfois 9h30. [...] Il y a des semaines où j'utilise la voiture tous les jours. Et quand le réservoir est vide, je la laisse. Disons, pour le mois, je peux venir en transport en commun deux ou trois fois. Car ça coûte cher, la voiture. Avec le prix de l'essence... j'en ai d'ailleurs parlé dernièrement avec le directeur pour qu'il essaye d'assouplir un peu [...] il y a des gens, il y a des collègues qui viennent en retard, parce qu'il y a des jours où on n'a pas assez de carburant dans le réservoir, donc on peut prendre les transports en commun ce jourlà, puisqu'il faut venir ici à 4h. (Extrait d'un entretien avec un salarié, décembre 2011)
\end{abstract}

Sommes-nous face à la création d'inégalités environnementales qui, dans ce contexte, constitueraient un renforcement des inégalités sociospatiales en raison d'une mesure environnementale ne prenant pas en compte les pratiques et le taux d'équipement automobile différenciés selon les populations? D'un point de vue subjectif, comme nous l'avons montré dans la deuxième partie de l'article, le dispositif ZAPA est considéré comme injuste et n'apparaît pas acceptable dans sa construction logique, puisque la grande majorité des personnes ayant participé à l'enquête pensent que les ménages pauvres seront les plus touchés. D’un point de vue plus objectif, s'appuyant sur la motorisation des ménages et la connaissance de leur mobilité, nous nous rendons compte que les conséquences sont plus complexes. Selon les réponses obtenues, les ménages dont les capacités financières sont faibles, mais qui ont un véhicule et en font un usage très rationalisé, s’adapteraient (Jouffe, 2007). Étant donné leur budget plus contraint, ils se rabattraient d'abord vers le transport en commun. Ils envisagent l'achat d'une autre automobile s'ils ne peuvent pas faire autrement, mais se questionnent sur leur capacité de réaliser cet achat dans un laps de temps réduit ${ }^{10}$. L’un des objectifs de la loi serait ainsi atteint (report modal, diminution de la circulation des voitures anciennes). Mais l’offre de transport collectif n’est pas nécessairement adaptée (lignes

10 De fait, le temps de renouvellement «naturel» des véhicules entre en ligne de compte dans les ménages pouvant se le permettre. Face à une obligation de le faire, ces mêmes ménages sont plus aptes à réaliser ce renouvellement plus rapidement que prévu, car ils l'auraient fait tout de même. 
surchargées, amplitude horaire de circulation ne correspondant pas à leur rythme de travail). En outre, cette adaptation se ferait au détriment de la qualité de vie de ces ménages, au contraire des salariés plus aisés, qui disposent plus souvent d'offres pour des mobilités différentes (plans de déplacement des entreprises, horaires "normaux») et ont un taux d'effort ${ }^{11}$ sur le logement et les transports proportionnellement moins important (Coutard et al., 2002; ADEME, 2008).

\section{Conclusion}

Le droit à l'environnement tend à devenir de plus en plus un droit-créance reconnu dans la hiérarchie des normes. Ainsi, la charte de l'Environnement en France (loi constitutionnelle $\mathrm{n}^{\circ} 2005-205$ du $1^{\mathrm{er}}$ mars 2005) énonce, dans son article premier, que «[c] hacun a le droit de vivre dans un environnement équilibré et respectueux de la santé», tandis que la jurisprudence établie sur ses bases s'étoffe (OGM - 2008564 DC 29/12/2008, par exemple). Pour autant, cette constitutionnalisation des droits et devoirs de la charte ne donne pas encore l'occasion de réfléchir sur le cumul des inégalités sociospatiales avec de possibles inégalités environnementales. Elle ne donne pas à réfléchir le juste et l'injuste dans la manière de concevoir l'action publique en matière d'environnement et, surtout, elle réduit l'environnement à des composantes écologiques, sans vision systémique et sans prendre en compte les aspects psychosociaux et l'ancrage spatial de l'environnement. La réflexion sur les inégalités environnementales nous y oblige pour comprendre ce qui est acceptable et désirable dans une société donnée, en prenant en compte le bien-être global des populations, mais aussi les différences qui existent entre elles.

Mettre en évidence et traiter les inégalités environnementales induit à ne pas édicter des mesures environnementales qui renforcent des disparités sociales et spatiales, et à travailler à éviter une surexposition des ménages précaires aux pollutions, risques et nuisances par rapport aux autres segments de la population. Plus encore, notre article cherche à démontrer qu'intégrer concomitamment aspects environnementaux et sociaux d'une mesure permettrait de rendre plus acceptables des démarches publiques et de mieux les appliquer. Le comportement contraire, comme c'est le cas ici, suscite méfiance et critiques et peut accroître les difficultés de certains types de population sans élever réellement le niveau de bien-être des autres.

Pour proroger cette réflexion, si une mesure des impacts socioenvironnementaux pèse plus fortement sur certaines populations, n’est-il pas important de réfléchir, très en amont de la décision incriminée, à la mise en place de dispositifs adéquats pour corriger des situations d'iniquité environnementale? Ainsi, afin de lutter contre les différentes formes d'iniquités (perçues et réelles) d'une ZAPA, les participants à l'enquête demandaient l'instauration de mesures de compensation générales (amélioration globale du système de transport, meilleure accessibilité pour les zones mal desservies) et spécifiques pour les plus précaires. Plus encore, ils souhaitaient être mieux associés à l'élaboration de ce type de dispositif qui ne prenait pas en compte la réalité de la pénibilité de leurs déplacements (justice distributive).

11 Rapport entre les dépenses réalisées par les ménages pour leur habitation principale et leur revenu disponible. 
Cependant, les compensations ne sont jamais réellement pensées simultanément au projet (Oberholzer-Gee et Weck-Hannemann, 2002). Elles sont toujours conçues comme des pansements éphémères visant à corriger une injustice momentanée, alors qu'elles s'inscrivent souvent dans des processus sociaux et spatiaux bien plus profonds. Or, les mesures compensatoires ne peuvent jouer leur rôle que si elles sont concertées et perçues comme des outils permettant de restaurer un équilibre social et environnemental pour tous (Gobert, 2010). Les ZAPA, telles qu'elles étaient appréhendées, nourrissaient une double ambiguïté: diriger l'action vers des publics cibles fragiles sans penser les mesures d'accompagnement. Elles s'avéraient en ce sens peu subtiles, car elles se réduisaient à une interdiction pour régler à court terme un problème systémique de pollution. D’où, aujourd'hui, l'enjeu fondamental pour les pouvoirs publics et les autres acteurs de mettre en place une stratégie plus efficace à court et à long termes qui repose sur une planification urbaine mieux coordonnée (Plan de protection de l'atmosphère, Plan climat énergie, Plan local d'urbanisme, etc.) et sur une plus grande sensibilisation des populations pour modifier, à terme, les comportements.

\section{Remerciements}

Cette enquête a été réalisée par ARISTAT avec Samira Ouardi et Xavier Zunigo, pour le compte de Plaine Commune. Nous remercions les trois évaluateurs anonymes de cet article qui nous ont permis de l'amender et d'en consolider la structure. 


\section{Bibliographie}

ADEME (2008) Le poids des dépenses énergétiques dans le budget des ménages en France. La lettre Ademe \& vous, $\mathrm{n}^{\circ} 11$.

BENBRAHIM-TALLAA, Lamia, BAAN, Robert A., GIROSSE, Yann, LAVBYSELRE, Béatrice, EL GHISSASSI, Fatima, BOURARES, Véronique, GUHA, Nada, LOOMIS, Sana et STRAVIF, Kurt (2012) Carcinogenicity of diesel-engine and gasoline-engine exhausts and some nitroarenes. The Lancet Oncology, vol.13, $n^{\circ} 7$, p. 663-664.

BOUDON, Raymond (1995) Le juste et le vrai. Paris, Fayard.

BRUNET, Philippe (2008) De l'usage raisonné de la notion de "concernement». Natures Sciences Sociétés, vol. 16, n 4, p. 317-325.

CHARDONNEL, Sonia et PAULHIAC, Florence (2012) La prise en compte des inégalités sociospatiales dans les politiques de mobilité: vers de nouvelles catégories de pensée et d'action. VertigO, $n^{\circ} 11$, [En ligne]. http://vertigo.revues. org/ 11738

CHARLES, Lionel (dir.) (2009) Pollutions atmosphériques et santéenvironnementale. Quels enjeux? Quels acteurs? Quelles préventions? Rapport PRIMEQUAL.

CHAUMEL, Marianne et LA BRANCHE, Stéphane (2008) Inégalités écologiques: vers quelle définition? Espace populations sociétés, $\mathrm{n}^{\circ} 1$. [En ligne]. http://eps. revues.org/index2418.html

CHEVALLIER, Maurice (2002) L'usage et l'accès à l'automobile: une liberté sous contraintes pour les personnes et ménages à faibles ressources. Rapport, programme Déplacements et inégalités PUCA-PREDIT.

COMMISSARIAT GÉNÉRAL AU DÉVELOPPEMENT DURABLE (CGDD) (2010) La mobilité des Français - Panorama issu de l'enquête nationale transports et déplacements. Paris, ministère de l’Écologie et du Développement durable.
COUTARD, Olivier, DUPUY, Gabriel et FOL, Sylvie (2002) La pauvreté périurbaine: dépendance locale ou dépendance automobile? Espaces et Sociétés, nos 108-109, p. 155-175.

DEGUEN, Séverine et ZMIROU-NAVIER, Denis (2010) Social inequalities resulting from health risks related to ambient air quality - A European review. European Journal of Public Health, vol. 20, $\mathrm{n}^{\circ}$, p. 27-35.

DIETZ, Simon et ATKINSON, Giles (2005) Public perceptions of equity in environmental policy: Traffic emissions policy in an english urban area. Local Environment: The International Journal of Justice and Sustainability, vol. 10, $\mathrm{n}^{\circ} 4$, p. 445-459.

DOBSON, Antony (1998) Justice and the environment: Conceptions of environmental sustainability and theories of distributive justice. Oxford University Press.

DUBET, François (2005) Propositions pour une syntaxe des sentiments de justice dans l'expérience de travail. Revue française de sociologie, vol. $46, n^{\circ} 3$, p. 495-528.

DUPUY, Gabriel (2006) La dépendance à l'égard de l'automobile. Predit, Transports recherche innovation, La Documentation française.

ECOLA, Liisa et LIGHT, Thomas (2010) Making congestion pricing equitable. Journal of Transportation Research Board, n²187, p. 53-59.

ELIASSON, Jonas et MATTSSON, Lars-Göran (2006) Equity effects of congestion pricing - Quantitative methodology and a case study for Stockholm. Transportation Research Part A, n 40 , p. 602-620.

EMELIANOFF, Cyria (2006) Connaître ou reconnaître les inégalités environnementales? ESO, Travaux et documents, $\mathrm{n}^{\circ} 25$, p. 35-43.

EVANS, Andrew.W. (1992) Road congestion pricing: When is it a good policy? Journal of Transport Economics and Policy, $\mathrm{n}^{\circ} 26$, p. 213-243. 
FLAMM, Michael (2004) Comprendre le choix modal. Les déterminants des pratiques modales et des représentations individuelles des moyens de transport. Lausanne, EPFL, thèse de doctorat non publiée.

FOL, Sylvie (2009) La mobilité des pauvres. Pratiques d'habitants et politiques publiques. Paris, Belin.

FRASER, Nancy (2005) Qu'est-ce que la justice sociale? Reconnaissance et redistribution. Paris, La Découverte.

GALLEZ, Caroline (2010) Les politiques de transports publics en France face à l'impératif de cohérence territoriale. Dans Hanja Maksi et Stéphanie Vincent (dir.) L'action publique face à la mobilité. Paris, L'Harmattan, p. 201-222.

GOBERT, Julie (2010) Les compensations socio-environnementales: un outil socio-politique d'acceptabilité de l'implantation ou de l'extension d'infrastructures? Université Paris Est, Créteil, thèse de doctorat non publiée.

GUEYMARD, Sandrine (2009) Inégalités environnementales en région Île-de-France: répartition sociospatiale des ressources, des handicaps et satisfaction environnementale des habitants. Université Paris 12 , Créteil, thèse de doctorat non publiée.

HAVARD, Sabrina, DEGUEN, Séverina, ZMIROU-NAVIER, Denis, SCHILLINGER, Charles et BARD, Denis (2009) Traffic-related air pollution and socio-economic status: A spatial autocorrelation study to assess environmental equity on a small-area scale. Epidemiology, vol.20, n² 2, p. 223-230.

INSTITUT NATIONAL DE LA STATISTIQUE ET DES ÉTUDES ÉCONOMIQUES (INSEE) (2009) Plaine Commune: un pôle d'emploi qui s'affirme entraînant de nombreux échanges domicile-travail. $\mathrm{N}^{\circ} 316$, p. 6.

JOUFFE, Yves (2007) Précaires mais mobiles. Tactiques de mobilité des travailleurs précaires flexibles et nouveaux services de mobilité. Université Paris-Est, Laboratoire LVMT, École nationale des ponts et chaussées, thèse de doctorat.
KAUFFMANN, Vincent, JEMELIN, Christophe et GUIDEZ, Jean-Marie (2001) Automobile et modes de vie urbains: quel degré de liberté? PREDIT, Transports recherche innovation, La Documentation française.

KAUFMANN, Vincent (2004) La mobilité comme capital? Dans Bertrand Montulet et Vincent Kaufmann (dir.) Mobilités, fluidités... libertés ? Bruxelles, Publication des Facultés universitaires Saint-Louis.

KELLERHALS, Jean et MODAK, Marianne (1997) Le sentiment de justice dans les relations sociales. Paris, Presses universitaires de France.

KNOEPFEL, Peter, LARRUE, Corinne et VARONE, Frédéric (2001) Analyse et pilotage des politiques publiques. Genève, Helbing et Lichtenbahn.

LA BRANCHE, Stéphane et CHARLES, Lionel (2012) Étude d'acceptabilité sociale de la ZAPA de l'agglomération grenobloise: synthèse des principaux résultats. Pollution Atmosphérique, $\mathrm{n}^{\circ}$ spécial, p. 27-231.

LAURENT Olivier et BARD, Denis (2007) Niveau socio-économique, pollution atmosphérique et santé: quelles approches méthodologiques? Extrapol, n³1, p. 3-7.

LAURIAN, Lucie (2008) Environmental injustice in France. Journal of Environmental Planning Management, vol. 51, n 1 , p. 55-79.

MORIN, Michel (2006) Pour une approche psycho-socio-environnementale des risques sanitaires. Dans Karine Weiss et Dorothée Marchand (dir.) Psychologie sociale de l'environnement. Rennes, Presses universitaires de Rennes.

OBERHOLZER-GEE, Felix et WECKHANNEMANN, Hannelore (2002) Pricing road use: Politico-economic and fairness considerations. Transportation Research Part D, $\mathrm{n}^{\circ}$ 7, p. 357-371.

ORFEUIL, Jean-Pierre (dir.) (2004) Transports, pauvretés, exclusions. Pouvoir bouger pour s'en sortir. Éditions de L'Aube. 
PASCAL, Laurence (2009) Effets à court terme de la pollution atmosphérique sur la mortalité. Revue française d'allergologie, vol. $49, n^{\circ} 6$, p. 466-476.

ROCCI, Anaïs (2007) De l'automobilité à la multimodalité? Analyse sociologique des freins et leviers au changement de comportements vers une réduction de l'usage de la voiture, Université Paris 5, thèse de doctorat.

SCHADE, Jens et SCHLAG, Bernhard (2003) Acceptability of urban transport pricing strategies. Transport Research Part F, $\mathrm{n}^{\circ}$ 6, p. 45-61.

SCHLOSBERG, David (2002) Environmental justice and the new pluralism. Oxford, Oxford University Press.

SMALL, Kenneth A. (1992) Using the revenues from congestion pricing. Transportation, vol. $19, \mathrm{n}^{\circ} 4$, p. 359-383.

STROH Emilie, OUDIN, Anna, GUSTAFSSON, Susanna, PILESJÖ, Petter, HARRIE, Lars, STRÖMBERG, Ulf et JAKOBSSON, Kristina (2005) Are associations between socio-economic characteristics and exposure to air pollution a question of study area size? An example from Scania. Sweden International Journal of Health Geography, n ${ }^{\circ}$, p. 30.

VIEGAS, José (2001) Making urban road pricing acceptable and effective: Searching for quality and equity in urban mobility. Transport Policy, vol. 8, n 4 , p. 289-294.

WALKER, Gordon, FAIRBURN, Jon, SMITH, Graham et MITCHELL, Gordon (2003) Environmental quality and social deprivation. Environment Agency, R\&D Technical Report.

WALSER, Martin (1983) Spheres of justice A defense of pluralism and equality. New York, Basic Books.

WENGLENSKI, Sandrine (2006) Regards sur la mobilité au travail des classes populaires. Une exploration du cas parisien. Les Cahiers Scientifiques du Transport, $\mathrm{n}^{\circ} 49$, p. 103-127.
WOLFF, Hendrik et PERRY, Lisa (2010) Trends in clean air legislation in Europe: Particulate matter and low emission zones. Review of Environmental Economic Policy, vol. 4, n² 2, p. 293-308. 